\title{
Re: Incidence and Risk Factors of Early Surgical Complications in Young Renal Transplant Recipients: A Persistent Challenge
}

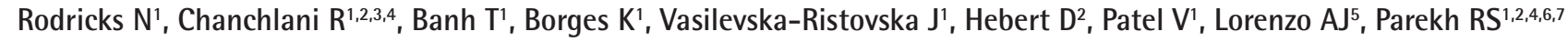 \\ 'The Hospital for Sick Children, Child Health Evaluative Sciences, Research Institute, Toronto, Canada \\ ${ }^{2}$ University of Toronto, the Hospital for Sick Children, Department of Pediatrics, Division of Nephrology, Toronto, Canada \\ ${ }^{3}$ McMaster University, McMaster Children Hospital, Department of Pediatrics, Division of Nephrology, Hamilton, Canada \\ ${ }^{4}$ University of Toronto, Institute of Health Policy, Management and Evaluation, Toronto, Canada \\ ${ }^{5}$ University of Toronto, the Hospital for Sick Children, Department of Surgery, Division of Urology, Toronto, Canada \\ ${ }^{6}$ University Health Network, Toronto, Canada \\ ${ }^{7}$ Dalla Lana School of Public Health, Toronto, Canada \\ Pediatr Transplant 2017. doi: 10.1111/petr.13006.
}

\section{EDITORIAL COMMENT}

Renal transplantation in children younger than 5 years of age is technically challenging and associated with increased mortality and graft loss. In this retrospective cohort study, the authors have examined the incidence and risk factors for early post transplant surgical complications in 83 recipients who were younger than 5 years old over three decades (1985-2014) which was divided into two periods. The incidence of surgical complications was $26.5 \%$ within 30 days post-transplant with vascular thrombosis being the most common complication in both periods. The number of complications did not significantly differ between two periods, however, the incidence rate of graft failure was higher in the first period. The authors have also highlighted the negative impact of early surgical complications on the graft and patient survival when compared to patients without complications. In a subgroup analysis of those with only vascular complications, children with abnormal coagulation profile were 3 times more prone to development of vascular complications, however, it was not significant. In this very young population, early surgical problems play a major role in graft failure and mortality. Kidney transplantation is still the best option for this very unique population in experienced centers. However, prevention of vascular complications require further optimization. Randomized controlled trials are required to determine the type, duration and benefits of prophylactic anticoagulant therapy in young children.

Yarkın Kamil Yakupoğlu, MD

๑Copyright 2017 by the Association of Urological Surgery / Journal of Urological Surgery published by Galenos Publishing House. 